\title{
Monitoring of High Slope and the Foundation of Security Forecast Model
}

\author{
Sifeng Zhang ${ }^{1, a}$, Weilin Zhang ${ }^{1, b}$, Yanmei $\mathrm{Li}^{2, \mathrm{c}}$ \\ ${ }^{1}$ School of Transportation Engineering, Shandong Jianzhu University, Jinan 250101, China; \\ ${ }^{2}$ Shandong Academy of Environmental Science, Jinan 250013, China \\ asddxzsf@163.com, ${ }^{\mathrm{b}}$ zwlsdjzu@163.com, ${ }^{\mathrm{c}} \mathrm{qqhrlym} @ 163 . c o m$
}

\begin{abstract}
Keywords: High slope; Slope monitoring; Security; Forecast model
Abstract: Dynamic in-situ monitoring is one of the important measures to ensure the stability of high slope engineering. Combined with a practical project, and based on the analysis of data that have been collected about the deformation of slope surface and interior and the stress state of support structures, the importance of dynamic monitoring has been exactly valued. On the basis of the analysis, a forecast model about slope security has been established which can offer an exemplification for the similar projects.
\end{abstract}

\section{Introduction}

Stability of high cut slope is an extremely complex non-linear, multi-parameter geotechnical problem. Studies on Stability of high cut slope is still at the level of laboratory and experience method [1]. There is a great difficulty in making accurate judgments of the safety of high cut slope in time and space. Especially, it is so difficult to grasp the steady state of the high cut slope having complex geological conditions and great potential hazards by theoretical analysis that dynamic monitoring system must be established [2,3]. In order to make the monitoring system fed back to the dynamic optimization design and information construction in geotechnical engineering timely, it seems to be particularly important to establish the forecast model of slope safety performance based on the information obtained from slope monitoring system.

High cut slope of an expressway in Shandong province is the highest cutting excavation, the largest excavation, the most complicated geological conditions and the toughest protection section in the whole line. Considering the special nature and importance of the project, the security forecast model that based on the complex three-dimensional monitoring facilities was established to monitor the construction safety, ensure the safety operation of the project and meet the project needs.

\section{Engineering Profile}

Engineering Geology Profile. The maximum excavation depth of the slope is about 80 meters. Exposed strata is limestone, marl and mudstone interbedding of the Majiagou Group of the Ordovician System. The cutting zone is also called "flood slope". There are faults behind the slope and the width is $2 \sim 20 \mathrm{~m}$. It is shown that the water level inside the slope is relatively stable and the slope mainly suffers the effect of hydrostatic pressure by monitoring the groundwater.

Design Overview of Slope. In this project, the partition management plan determined by the principles of the comprehensive management of landslides and cutting's poor geological conditions, which include that in the upper area of the landslide, setting steps, cutting loose slope by the proportion of 1:1 and reducing the weight of slope, and deep unstable rock reinforced by prestressed anchor cable. Full-length bond anchor and netting sprayed concrete were used to reinforce a certain depth of broken rock on the surface, improve the integrity and strength of the slope. Slope drainage with the method of horizontal dewatering holes, and anti-slide pile was set to block the slide at the toe of the slope.

Design of Monitoring System. The monitoring system consists of deformation monitoring and stress monitoring. The main monitor contents are as follows: (1) Deformation monitoring: the deformation of the potential sliding surface and the main sliding zone in the slope was monitored by the borehole clinometers through digging deep holes and measuring its tilt. (2) Anchor cable prestressed monitoring: anchor dynamometer was buried between anchorage device and steel 
backing plate to monitor anchor cable prestress and its changes. (3) Anti-slide pile pressure monitoring: soil pressure cells were buried between the pile back and the mountain to monitor the pile's soil pressure from the slope body. (4) Slope hydrodynamic pressure monitoring: drilling and burying pore water pressure gauge in the main slide area to monitor the change of pore water pressure. The cross section's arrangement of the specific observation instruments is shown in figure 1.

\section{Application of dynamic monitoring in this project}

Application of borehole clinometers. As one of the main instruments for the monitoring of geotechnical engineering, the borehole clinometer is generally used to determine the position of the potential slip surface of the slope or observe the slide of the sliding surface. In this project, a total of seven clinometers were buried, and distributed on all levels of the steps. CX3 was located in the middle of the slope, which suffered the biggest influence. The curves of the changes in each period are plotted in the following figure 2 .

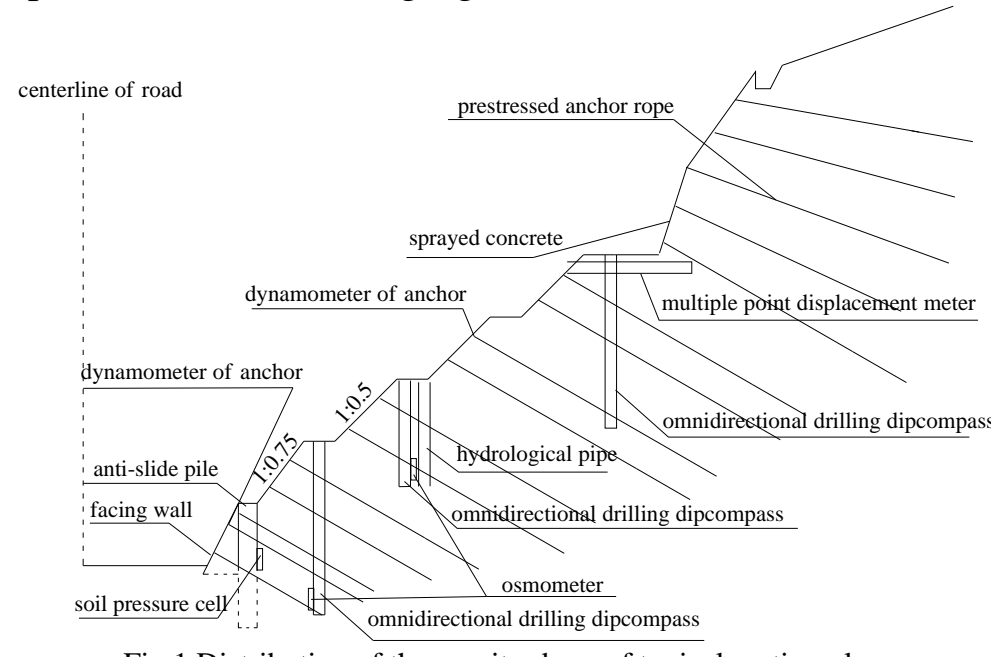

Fig.1 Distribution of the monitor bore of typical section plane

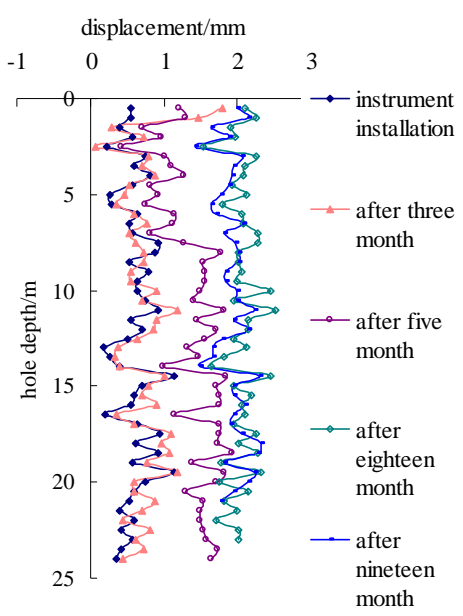

Fig.2 Fluctuation curve of displacement-depth

As we can see, from within 5 months after the instrument embedment, too fast excavation speed of the last step of the slope and the blasting excavation of the anti-slide pile construction in the slope toe made the average displacement of the slope reach to $1.0-1.2 \mathrm{~mm}$, which caused a great threat to the stability of the slope. Therefore, the construction unit adjusted the construction program, slowed the speed of slope excavation and built up support to avoid the tendency of excessive slip timely. In addition, it can be seen from the figure that the displacement mutation phenomenon was more obvious in the depth of $15 \mathrm{~m}$, indicating that the slope affected by Marl interlayer has mobile tendency.

Application of anchor dynamometer. The change of the prestressed anchor most directly and rapidly reflects the stress state of the slope. Just as can be seen from figure 3, the impact that anchor cable suffered during construction is the greatest. Because of the slope excavation unloading, rock mass stress can be released, so that the prestress value increased, which is corresponding to the clinometer monitoring results. Thereby it is particularly important to control the speed of excavation in construction and avoid the anchor being pulled out because of excessive prestress.

After the completion of the construction of the slope, rainfall is the biggest factor of affecting the prestress value. Because of the rain's erosion, rock mass softening, strength reduction, combined with the effect of hydrodynamic pressure, it will inevitably lead to downward tendency, which will lead to the increase of prestress value if the water in the slope body can not be discharged in time. In addition, it can be seen from figure 4, it is not synchronous between the increase of prestress and the occurrence of rainfall because the infiltration of rainwater will take some time. After the crack water gradually dissipated, the prestress value will resume the original level [4]. In order to avoid the effect of cyclic loading, the waterproof and drainage of the slope is 
very important before the rainy season.

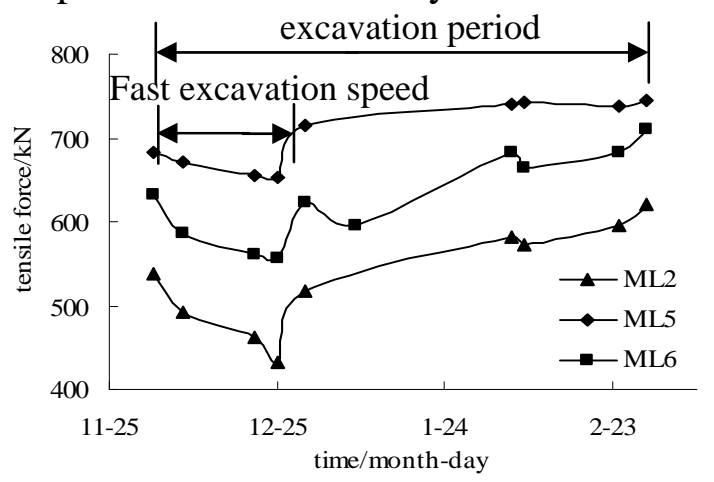

Fig.3 Increase of prestress value due to excavation

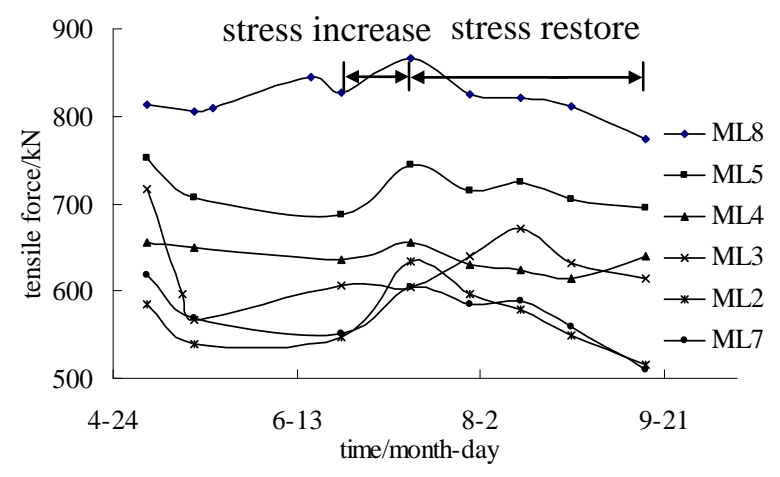

Fig.4 Influence on prestress value due to rainfall

Application of soil pressure gauge. The soil pressure gauge that buried between the anti-slide pile and the slope is very important for monitoring the change of displacement in slope toe. The value of the soil pressure gauge reflects the action of sliding body against anti-slide pile, and has a great influence on the reliability of anti-slide pile.

Figure 5 is the curve of the monitoring value of a soil pressure gauge. As can be seen from the figure, the pressure increased significantly in a period of the time before and after the completion of support, which was related to the slope displacement, and coincided with the change of the anchor dynamometer and the borehole clinometer. After the completion of the slope reinforcement, the pressure value of most soil pressure gauges began to decrease, which indicated that the safety degree of the slope had been greatly improved, and the effect of slope reinforcement was obvious. However, a large number of rainfall kept in the slope body because of the continuous rainfall(5-8 months), and blocked the pipes in the central part of the mountain, which caused the soil pressure in the middle of the slope to reach the maximum value in September. To this end, the construction unit encrypted the monitoring frequency and artificial patrol, and timely cleared the drainage system of the slope. After the end of the rainy season, the amount of displacement did not continue to increase significantly, indicating that the drainage situation of the slope has been improved.

Applications of osmometer. The stability of the slope can be analyzed by measuring the pore water pressure and its dissipation. Figure 6 is a curve of different osmometer's pressure with different time. As can be seen from the figure, the difference of the local geological conditions of the slope caused poor drainage in local location and pressure values higher than other parts, which is most obvious in the middle of the slope.

\section{Establishment of the security forecast model of slope}

Obviously, only relying on a certain monitoring data can't evaluate the stability of the high and steep slope or predict the high slope's sliding range and sliding time. Various monitoring data must be collected and analyzed to make the evaluation or give warning and forecast about the overall

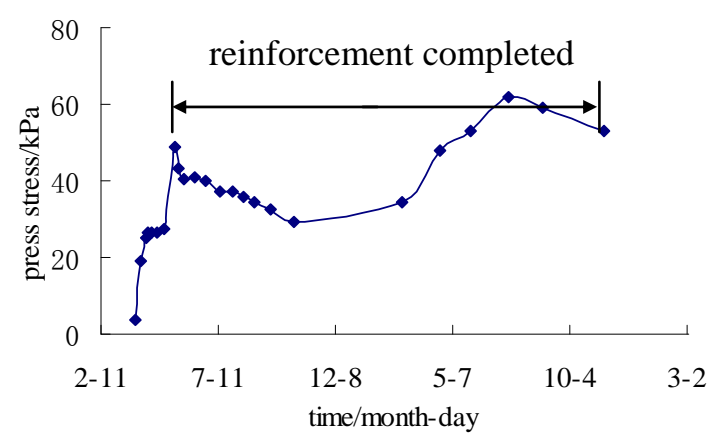

Fig. 5 Fluctuation curve of soil press

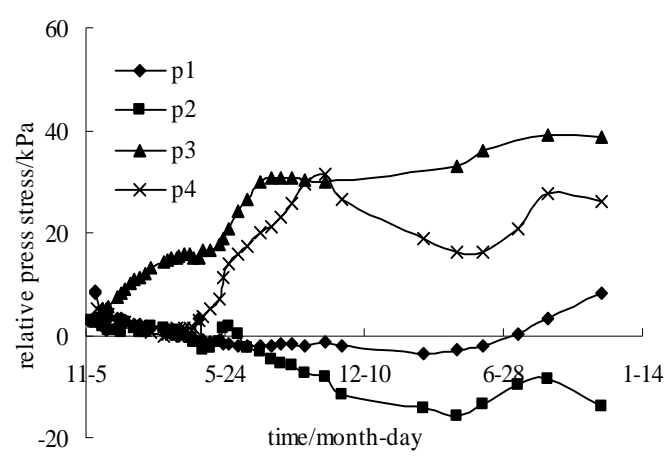

Fig.6 Fluctuation curve of pore water pressure

stability of the slope. However, even with a lot of observational data, it is still difficult to make a 
direct evaluation and prediction of the security situation of the building. The establishment of the correlation among the observational data is extremely complex and difficult [5].

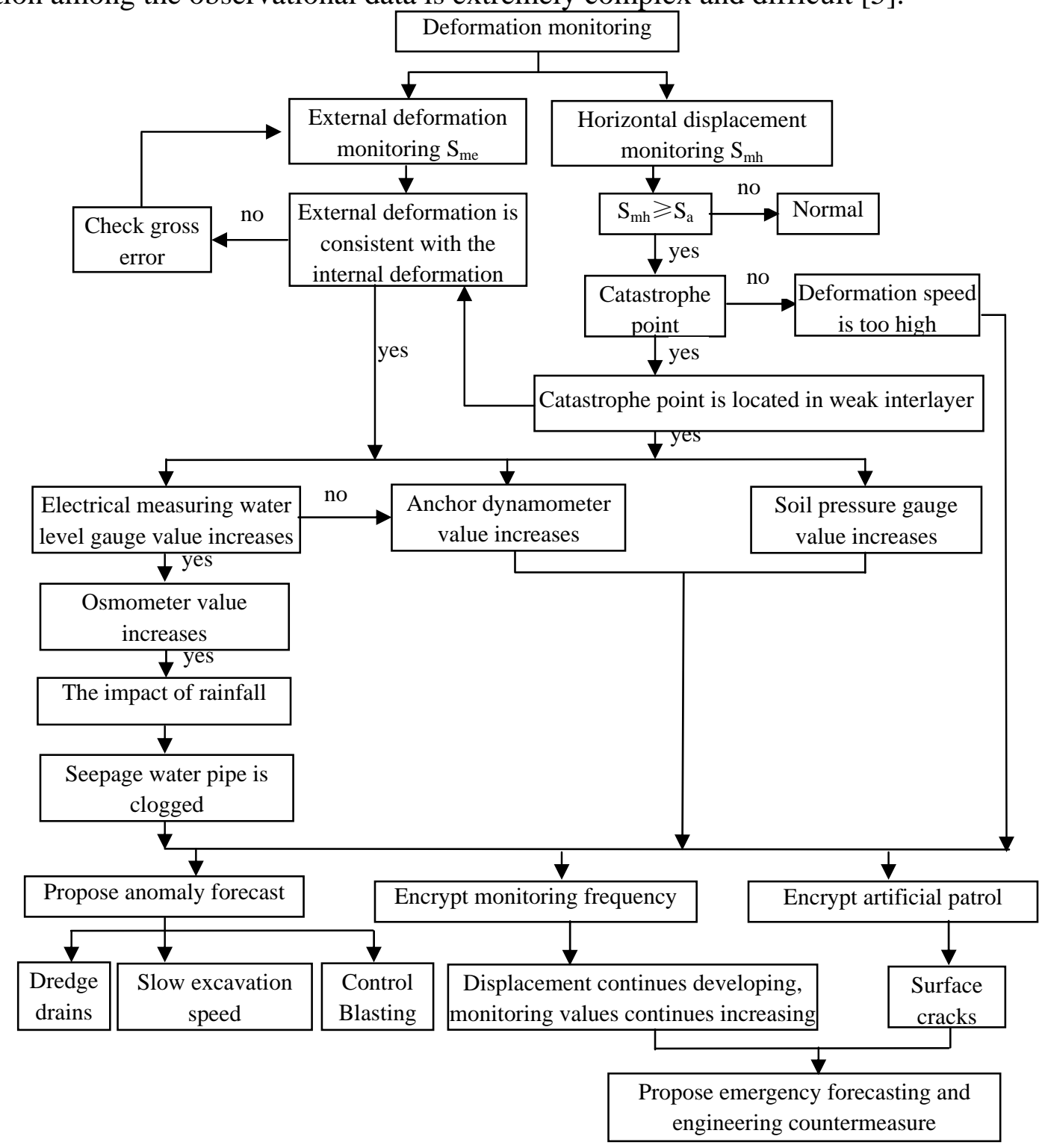

Fig.7 Forecast model of slope safety performance

On the basis of the implementation of a high slope monitoring system, this paper summarizes and analyzes the relationship between the numerical variation of the monitoring instruments and the overall stability of the slope. On the basis of reference of other literature [6], the forecast model of slope safety performance was put forward (as shown in figure 7), which was shown to be effective by the high slope practice. The security forecast model of slope has guiding significance for the design and prediction of similar projects, and can offer an exemplification for the similar projects.

\section{Conclusions}

According to the analysis of this paper, we can know that establishing a complete monitoring system on the slope, especially the high and steep slope, can accurately obtain the stability information of slope. Under the premise to ensure the safety of the slope, establishing a complete monitoring system on the slope can improve the construction efficiency, speed up the construction progress. We can take corresponding measures to ensure the safety and stability of the slope and avoid unnecessary economic losses if abnormal phenomenon appears. Therefore, slope security monitoring is an integral part of the construction, which is necessary for finding out the working characteristics of the high slope and verifying the design. 
In this paper, the security forecast model of slope based on the actual slope engineering monitoring method can offer an exemplification for the monitoring design of similar slope.

\section{Acknowledgments}

This work was financially supported by the National Natural Science Foundation of China (No. 41372300 \& 51108252).

\section{References}

[1] Wang Hao, Liu Lijun, Liu Baohua. Application of RTK technology to slope displacement monitoring in Baiyinhua open mine[J]. Coal Technology, 2009,28(11):106-108.(in chinese)

[2] Zhang Zhiying, He Kun. A study on slope monitoring system and methods[J]. Soil Engineering and Foundation, 2006,20(3):82-84.(in chinese)

[3] Shi Kejun, Chen Weihong. Safety supervision on the exit slope of right waterway of Longtan Hydropower Station during construction period[J]. Water Power, 2003(10):91-94.(in chinese)

[4] Jing Feng, Zhu Jiebing, Bian Zhihua, Chen Hao. Analysis on the prestressing variety characteristics of prestressed cable in soft rock slope[J]. Chinese Journal of Underground Space and Engineering, 2008,4(5):820-824.(in chinese)

[5] Zhu Xiaowen, Zhao Qilin, Yue Jianping, Ji Lin. Monitoring system of long-span suspension bridge foundation[J]. Journal of Highway and Transportation Research and Development, 2005,22(6):98-107.(in chinese)

[6] Chen Zhijian. Theory and key technology of monitoring and evaluation model establishment on layered rocky slope engineering[J]. Chinese Journal of Rock Mechanics and Engineering, 2003,22(12).(in chinese) 